\title{
Um Algoritmo Aritmético para Análise Espectral Não-Uniforme
}

\author{
Juliano B. Lima, Ricardo M. Campello de Souza e Hélio M. de Oliveira
}

\begin{abstract}
Resumo-Este artigo apresenta uma extensão da transformada aritmética de Fourier que permite analisar o espectro de um sinal discreto em freqüências arbitrárias. $O$ procedimento proposto, que está relacionado à transformada discreta de Fourier não-uniforme, possui características atrativas em termos de complexidade computacional. Resultados de simulações e cenários de aplicação são discutidos.
\end{abstract}

Palavras-Chave-Análise espectral, transformada aritmética de Fourier, transformada discreta de Fourier não-uniforme.

Abstract-This paper presents an extension of the arithmetic Fourier transform which allows to perform the spectral analysis of a discrete-time signal in arbitrary frequencies. The proposed procedure, which is related to the nonuniform discrete Fourier transform, has attractive characteristics in terms of computational complexity. Results of simulations and application scenarios are discussed.

Keywords-Spectral analysis, arithmetic Fourier transform, nonuniform discrete Fourier transform.

\section{INTRODUÇÃO}

As ferramentas matemáticas para análise espectral de sinais possuem extrema importância em diversas aplicacões de Engenharia. Quando se fala em analisar sinais discretos, a transformada discreta de Fourier (DFT) é, sem dúvida, a técnica mais conhecida e utilizada, sendo importante, particularmente, no projeto de filtros para modificação das características de um sinal e para detecção de tons [1], [2], [3].

Em muitos cenários, entretanto, o fato da DFT amostrar o espectro de forma equispaçada compromete, em termos de precisão, a sua aplicabilidade direta. Quando se calcula a DFT de uma sequiência $x[i]$, com $N$ pontos obtidos pela amostragem de um sinal contínuo a uma freqüência $f_{s}$, apenas as freqüências $f_{k}=k \frac{f_{s}}{N}, k=0,1, \ldots, N-1$, podem ser analisadas com precisão. Isso equivale a avaliar $X(z)$, a transformada $z$ de $x[i]$, em $N$ ângulos igualmente espaçados sobre a circunferência unitária no plano $z$.

Para eliminar a restrição descrita, Bagchi e Mitra propuseram a transformada discreta de Fourier não-uniforme (NDFT) [2] que, avaliando $X(z)$ em pontos arbitrários, possibilita análise espectral em outras freqüências. Usualmente, a NDFT é implementada através do algoritmo de Goertzel, que é eficiente em situações em que apenas algumas componentes de um sinal precisam ser calculadas.

O presente artigo propõe o cálculo da NDFT através da transformada aritmética de Fourier (AFT). Originalmente, a

Juliano B. Lima, Ricardo M. Campello de Souza e Hélio M. de Oliveira Departamento de Eletrônica e Sistemas, Centro de Tecnologia e Geociências, Universidade Federal de Pernambuco, Recife, Brasil, E-mails: juliano_bandeira@ieee.org,ricardo@ufpe.br,hmo@ufpe.br
AFT foi introduzida como um algoritmo para a obtenção dos coeficientes da série de Fourier de um sinal contínuo, sendo, posteriormente, adaptada para o cálculo da DFT [4], [5], [6]. Aqui, dois procedimentos para o cálculo da NDFT via AFT são apresentados. A principal motivação para o desenvolvimento de tais procedimentos é a baixa complexidade aritmética envolvida na implementação da AFT, que é, na maioria dos casos, mais eficiente que o algoritmo de Goertzel.

Este artigo está organizado como segue. Na Seção II, uma breve revisão da teoria da transformada aritmética de Fourier é apresentada. Na Seção III, dois procedimentos para o cálculo da NDFT via AFT são introduzidos. Na Seção IV, um cenário de aplicação da AFT à análise espectral não-uniforme é estudado. Resultados de diversas implementações da AFT aplicáveis ao cenário proposto são discutidos. Na Seção V, são apresentadas as conclusões desse trabalho.

\section{A TRAnSFormadA ARITMÉticA DE Fourier}

A possibilidade de expressar qualquer função periódica através de uma série de Fourier representou o primeiro passo no desenvolvimento da teoria para análise de sinais. Na forma trigonométrica, como é mais conhecida, a série de Fourier de um sinal contínuo $x(t)$, com período $T$ e banda limitada, é escrito sob a forma:

$$
x(t)=a_{0}+\sum_{k=1}^{N}\left[a_{k} \cos (2 \pi k t / T)+b_{k} \sin (2 \pi k t / T)\right] .
$$

Na equação acima, $a_{k}$ e $b_{k}$ são denominados coeficientes harmônicos e, classicamente, sua obtenção requer o cálculo de integrais. Em 1903, H. Bruns desenvolveu um procedimento para computar $a_{k}$ e $b_{k}$ usando a fórmula de inversão de Möbius para séries finitas [7]. Diferentemente do método clássico, apenas adições e algumas multiplicações por fatores de escala são necessárias, o que envolve complexidade computacional relativamente baixa.

Posteriormente, o procedimento proposto por Bruns recebeu o nome de transformada aritmética de Fourier (AFT), técnica que foi redescoberta e revisitada em diversos outros trabalhos. Dentre as diferentes versões da AFT [8], a mais eficiente em termos de complexidade computacional é a proposta em [4]. Esse algoritmo, chamado AFT simplificada, é resumido na definição e no teorema que seguem.

Definição 1 (Médias de Bruns): A $2 k$-ésima média alternante de Bruns, $B_{2 k}(\alpha)$, relacionada a um sinal $x(t)$, com período $T$, é definida por

$$
B_{2 k}(\alpha)=\frac{1}{2 k} \sum_{m=0}^{2 k-1}(-1)^{m} x\left(m \frac{T}{2 k}+\alpha T\right) .
$$


Teorema 1 (AFT simplificada): Os coeficientes harmônicos $a_{k}$ e $b_{k}$ da série de Fourier, com $N$ termos, de um sinal $x(t)$, com período $T$ e banda limitada, são dados por [4]

$$
\begin{aligned}
a_{0} & =\frac{1}{T} \int_{0}^{T} x(t) d t \\
a_{k} & =\sum_{l=1,3, \ldots}^{\lfloor N / k\rfloor} \mu(l) B_{2 k l}(0), \\
b_{k} & =\sum_{l=1,3, \ldots}^{\lfloor N / k\rfloor} \mu(l)(-1)^{\frac{(l-1)}{2}} B_{2 k l}\left(\frac{1}{4 k l}\right),
\end{aligned}
$$

onde $k=1, \ldots, N, \mu($.$) é a função de Möbius e B_{2 k}($.$) é a$ $2 k$-ésima média alternante de Bruns.

$\mathrm{O}$ cálculo de todos os coeficientes $a_{k}$ e $b_{k}$ pelas equações acima requer $N$ multiplicações reais e cerca de $N^{2} / 2$ adições reais [4].

\section{A. A Transformada Discreta de Fourier Via AFT}

A transformada aritmética de Fourier torna-se particularmente interessante quando aplicada a sinais discretos. Nesse contexto, é possível calcular os coeficientes da transformada discreta de Fourier (DFT) de uma seqüência $x[i], \operatorname{com} N$ pontos, que é definida por [1]

$$
X[k]=\sum_{i=0}^{N-1} x[i] e^{-\frac{j 2 \pi k i}{N}} .
$$

A DFT inversa é obtida por

$$
x[i]=\frac{1}{N} \sum_{k=0}^{N-1} X[k] e^{\frac{j 2 \pi k i}{N}} .
$$

Como a DFT de uma seqüência complexa pode ser obtida a partir das transformadas de duas seqüências reais, sem perda de generalidade, considera-se $x[i]$ uma seqüência real. Empregando a representação trigonométrica para a exponencial complexa e efetuando a substituição $X[k]=R[k]+j I[k]$, onde $R[k]$ e $I[k]$ representam, respectivamente, a parte real e a parte imaginária de $X[k]$, reorganiza-se a última equação e obtém-se [5]

$$
x[i]=\frac{R_{0}}{N}+\frac{2}{N} \sum_{k=1}^{\frac{N}{2}}\left[R[k] \cos \left(\frac{2 \pi k i}{N}\right)-I[k] \sin \left(\frac{2 \pi k i}{N}\right)\right] .
$$

Realizando uma comparação direta entre a equação acima e a Equação (1), identificam-se as relações

$$
a_{k}=\frac{2 R[k]}{N} \quad \text { e } \quad b_{k}=-\frac{2 I[k]}{N} .
$$

para $k=1,2, \ldots, N / 2$. Adicionalmente, o período $T$ é trocado pelo comprimento $N$ e, no limite do somatório, $N$ é substituído por $N / 2$. Desse modo, as médias de Bruns são redefinidas e o Teorema 2 é enunciado.

Definição 2: A $2 k$-ésima média alternante de Bruns, $\tilde{B}_{2 k}(\alpha)$, relacionada a uma seqüência $x[i]$, com $N$ pontos, é definida por

$$
\tilde{B}_{2 k}(\alpha)=\frac{1}{2 k} \sum_{m=0}^{2 k-1}(-1)^{m} x\left[m \frac{N}{2 k}+\alpha N\right] .
$$

Teorema 2: As componentes $R[k]$ e $I[k]$ de $X[k]=R[k]+$ $j I[k], k=1, \ldots, N / 2$, da transformada discreta de Fourier de uma seqüência real $x[i]$, com $N$ pontos, são calculadas por

$$
\begin{aligned}
& R[k]=\frac{N}{2} \sum_{l=1,3, \ldots}^{\left\lfloor\frac{N}{2 k}\right\rfloor} \mu(l) \tilde{B}_{2 k l}(0), \\
& I[k]=-\frac{N}{2} \sum_{l=1,3, \ldots}^{\left\lfloor\frac{N}{2 k}\right\rfloor} \mu(l)(-1)^{\frac{(l-1)}{2}} \tilde{B}_{2 k l}\left(\frac{1}{4 k l}\right) .
\end{aligned}
$$

As componentes $X[k], k=N / 2+1, \ldots, N-1$, são obtidas por simetria. De forma análoga à AFT simplificada original, a obtenção de todas as componentes $X[k]$ envolve $N / 2$ multiplicações e cerca de $N^{2} / 8$ adições.

\section{A TRANSFORMADA DiscretA DE FOURIER NÃO-UNIFORME VIA AFT}

\section{A. A Transformada Discreta de Fourier Não-Uniforme}

A transformada discreta de Fourier não-uniforme de uma seqüência $x[i]$, de comprimento $N$, é definida como

$$
X\left(z_{k}\right)=\sum_{i=0}^{N-1} x[i] z_{k}^{-i}, k=0,1, \ldots, N-1,
$$

onde $z_{0}, z_{1}, \ldots, z_{N-1}$ são pontos distintos localizados arbitrariamente no plano $z$. O cálculo da NDFT de um vetor $\mathbf{x}$ é escrito como uma multiplicação matricial

$$
\mathbf{X}=\mathbf{D} \mathbf{x} \text {. }
$$

A NDFT inversa é calculada com o uso da matriz $\mathbf{D}^{-1}$, cuja existência é assegurada e cuja obtenção envolve $O\left(N^{3}\right)$ operações [2].

Quando se deseja simplesmente avaliar o espectro de um sinal em freqüências não amostradas por uma DFT, um caso particular da NDFT pode ser considerado. Para isso, introduzse o parâmetro $\beta, 0<\beta<1$, responsável por deslocar os pontos que, na freqüência, seriam amostrados por uma DFT; na Equação (8), basta escolher $z_{k}=e^{\frac{j 2 \pi(k-\beta)}{N}}$. Isso resulta na definição a seguir.

Definição 3: A transformada discreta de Fourier nãouniforme de uma seqüência $x[i], i=0,1, \ldots, N-1$, é a seqüência $X[k], k=0,1, \ldots, N-1$, cujas componentes são calculadas por

$$
X[k]=\sum_{i=0}^{N-1} x[i] e^{\frac{-j 2 \pi(k-\beta) i}{N}}, k=0,1, \ldots, N-1 .
$$

Teorema 3: A transformada discreta de Fourier nãouniforme inversa de uma seqüência $X[k], k=0,1, \ldots, N-1$, é a seqüência $x[i], i=0,1, \ldots, N-1$, cujas componentes são calculadas por

$$
x[i]=\frac{1}{N} \sum_{k=0}^{N-1} X[k] e^{\frac{j 2 \pi(k-\beta) i}{N}}, i=0,1, \ldots, N-1 .
$$

Demonstração: A prova desse teorema parte da substituição da Equação (9), com o índice $i$ trocado por $r$, na Equação (10). 
Desse ponto em diante, o termo transformada discreta de Fourier não-uniforme refere-se à definição apresentada na Equação (9). O exemplo a seguir ilustra sua aplicabilidade.

Exemplo 1: Deseja-se analisar a freqüência $f=2062,5 \mathrm{~Hz}$ num sinal $x[i]$, de comprimento $N=64$, obtido mediante amostragem de um sinal contínuo $x(t)$ à freqüência $f_{s}=$ $8000 \mathrm{~Hz}$. A componente espectral associada a $f$ é dada por

$$
k-\beta=f \frac{N}{f_{s}}=2062,5 \frac{64}{8000}=16,5 .
$$

Portanto, na Equação (9), calcula-se $X[17]$, com $\beta=0,5$.

A forma mais eficiente de realizar o cálculo propriamente dito de uma componente $X[k]$, como no exemplo acima, é através do algoritmo de Goertzel. Para cada componente calculada, são necessárias $M_{G}(N)$ multiplicações e $A_{G}(N)$ adições reais, dadas por [3]

$$
\begin{aligned}
M_{G}(N) & =N+4, \\
A_{G}(N) & =2 N+2 .
\end{aligned}
$$

\section{B. NDFT Via AFT}

Nesta subseção, mostra-se como as componentes da transformada discreta de Fourier não-uniforme de uma seqüência podem ser calculadas através da AFT. Duas abordagens são propostas. A primeira delas consiste, basicamente, em modificar a sequiência $x[i]$, cuja NDFT deve ser calculada, e gerar a seqüência $\hat{x}[i]$. A AFT é aplicada a $\hat{x}[i]$ conforme descrito na Seção II-A. Na segunda abordagem, a expressão para o cálculo das médias de Bruns é alterada e a AFT é, então, aplicada à própria seqüência $x[i]$.

1) Abordagem 1 ( $\left.A F T_{1}\right)$ : Como ponto de partida, reescreve-se a Equação (10), que expressa a NDFT inversa, como

$$
x[i]=\frac{1}{N} e^{-\frac{j 2 \pi \beta i}{N}} \sum_{k=0}^{N-1} X[k] e^{\frac{j 2 \pi k i}{N}}, i=0,1, \ldots, N-1 .
$$

Daí, define-se a seqüência

$$
\hat{x}[i]=x[i] e^{\frac{j 2 \pi \beta i}{N}}=\frac{1}{N} \sum_{k=0}^{N-1} X[k] e^{\frac{j 2 \pi k i}{N}}, i=0,1, \ldots, N-1 .
$$

Observando a equação acima, percebe-se que $X[k], k=$ $0,1, \ldots, N-1$, equivale à DFT de $\hat{x}[i], i=0,1, \ldots, N-1$. Esta última sequiência é, entretanto, complexa, podendo ser escrita como $\hat{x}[i]=\hat{x}_{R}[i]+j \hat{x}_{I}[i]$, onde $\hat{x}_{R}[i]$ e $\hat{x}_{I}[i]$ denotam, respectivamente, a parte real e a parte imaginária de $\hat{x}[i]$. Nessas condições, aplica-se a AFT separadamente a $\hat{x}_{R}[i]$ e a $\hat{x}_{I}[i]$. Como resultado, obtém-se as seqüências $\hat{X}_{R}[k]$ e $\hat{X}_{I}[k]$, que compõem $X[k]=\hat{X}_{R}[k]+j \hat{X}_{I}[k]$.

Um fator que permite avaliar a praticidade do procedimento descrito é a complexidade aritmética que o mesmo demanda. Assim, com o objetivo de realizar uma comparação com o algoritmo de Goertzel, determina-se o número de multiplicações e o de adições reais envolvidas na obtenção de uma componente específica da NDFT via AFT.

A obtenção de uma componente $\hat{X}_{R}[k]$ requer, no cálculo das médias de Bruns, $2\lfloor(N / 2 k+1) / 2\rfloor$ multiplicações por fatores de escala, as quais podem incorporar as multiplicações por $N / 2$ nas Equações (6) e (7). Para somar as médias, $2(\lfloor(N / 2 k+1) / 2\rfloor-1)$ adições são necessárias; $2 \sum_{l=1,3, \ldots}^{\lfloor N / 2 k\rfloor}(2 l k-1)$ adições de amostras são requeridas para obter todas as médias. Esses mesmos números de multiplicações e adições estão envolvidos no cálculo de $\hat{X}_{I}[k]$. A obtenção de $X[k]$ por $\hat{X}_{R}[k]$ e $\hat{X}_{I}[k]$ requer mais 2 adições. Assim, o número total de multiplicações e o de adições requeridos para o cálculo de uma componente $X[k]$ são dados, respectivamente, por

$$
\begin{aligned}
& M_{1}(N, k)=4\left\lfloor\frac{N+k}{2 k}\right\rfloor, \\
& A_{1}(N, k)=4\left(\left\lfloor\frac{N-k}{2 k}\right\rfloor+\sum_{l=1,3, \ldots}^{\left\lfloor\frac{N}{2 k}\right\rfloor}(2 l k-1)+\frac{1}{2}\right) .
\end{aligned}
$$

A complexidade aritmética determinada pelas Equações acima é consideravelmente menor que aquela associada ao algoritmo de Goertzel. No entanto, para que a AFT seja aplicada, é necessário obter as amostras $\hat{x}[i]$ usadas no cálculo das médias de Bruns. Numa situação extrema, em que se precise obter $\hat{x}[i]$ para $i=0,1, \ldots, N-1$, cerca de $2 N$ multiplicações reais extras seriam necessárias. Isso tornaria a AFT ineficiente, quando comparada ao algoritmo de Goertzel.

A estratégia para diminuir o número de operações requeridas por essa abordagem é, então, considerar apenas os termos em que $l=1$ nos somatórios das Equações (6) e (7). Esses termos estão associados a uma freqüência específica $f$. Os termos em que $l=3$, por sua vez, estão associados à freqüência $3 f$ e serão significativos apenas se essa frequiência estiver presente no sinal analisado. Fato análogo acontece para os termos em que $l \geq 5$ ( $l$ ímpar). Em cenários em que é possível a presença e é necessária a análise de apenas algumas componentes espectrais, a condição descrita é facilmente respeitada, tornando, portanto, a consideração inicial válida.

Assumindo a realização do truncamento descrito acima, $8 k$ multiplicações são requeridas na obtenção de amostras $\hat{x}[i]$ necessárias ao cálculo das 4 médias de Bruns mais significativas. Seguindo um raciocínio análogo ao desenvolvido para derivar as Equações (11) e (12), chega-se ao número total de multiplicações, $M_{1, T}(N, k)$, e ao de adições, $A_{1, T}(N, k)$, para se obter uma componente $X[k]$, que são dados por

$$
\begin{aligned}
M_{1, T}(N, k) & =8 k+4, \\
A_{1, T}(N, k) & =8 k-2 .
\end{aligned}
$$

Nas Equações acima, o subscrito $T$ refere-se ao truncamento realizado.

2) Abordagem $2\left(A F T_{2}\right)$ : É possível calcular componentes $X[k]$ da NDFT de $x[i]$ a partir de uma modificação na Definição 2. Sendo $f$ a freqüência que se deseja analisar e considerando o cenário descrito no Exemplo 1, os parâmetros $k$ e $\beta$ são obtidos por

$$
k-\beta=f \frac{N}{f_{s}} .
$$

Após algumas manipulações, a última equação é reescrita como

$$
k=f \frac{N}{\left(\frac{f N f_{s}}{f N+f_{s} \beta}\right)}=f \frac{N}{f_{s}^{\prime}} .
$$


Observando a última expressão, pode-se afirmar que, caso o sinal $x(t)$ tivesse sido amostrado a uma freqüência $f_{s}^{\prime}$, produzindo uma seqüência $\bar{x}[i], i=0,1, \ldots, N-1$, a freqüência $f$ estaria associada à componente $\bar{X}[k]$ da DFT dessa sequiência. É como se o ajuste $\beta$, em vez de ter sido aplicado à amostragem espectral, como anteriormente, tivesse sido incorporado à freqüência de amostragem usada no processo de discretização de $x(t)$. Assim, o cálculo de $\bar{X}[k]=\bar{R}[k]+j \bar{I}[k]$ implicaria usar as Equações (6) e (7), que incluiriam médias de Bruns computadas a partir de

$$
\tilde{B}_{2 k}(\alpha)=\frac{1}{2 k} \sum_{m=0}^{2 k-1}(-1)^{m} \bar{x}\left[m \frac{N}{2 k}+\alpha N\right] .
$$

Como não se dispõe da sequiência $\bar{x}[i]$, usam-se as relações $x[i]=x\left(i / f_{s}\right)$ e $\bar{x}[i]=x\left(i / f_{s}^{\prime}\right)$ para verificar que

$$
\bar{x}[i]=x\left[i\left(1+\frac{\beta f_{s}}{f N}\right)\right] \text {. }
$$

Substituindo o resultado da relação acima na Equação (13), obtém-se a seguinte expressão para as médias de Bruns:

$$
\tilde{B}_{2 k}(\alpha)=\frac{1}{2 k} \sum_{m=0}^{2 k-1}(-1)^{m} x\left[\left(m \frac{N}{2 k}+\alpha N\right)\left(1+\frac{\beta f_{s}}{f N}\right)\right] .
$$

Utilizando essa abordagem, a análise espectral com precisão da freqüência desejada é factível, não havendo necessidade de se obter qualquer componente da seqüência $\hat{x}[i]$, anteriormente introduzida. O número de multiplicações, $M_{2}(N, k)$, e o de adições, $A_{2}(N, k)$, envolvidas na presente abordagem, são

$$
\begin{aligned}
& M_{2}(N, k)=2\left\lfloor\frac{N+k}{2 k}\right\rfloor, \\
& A_{2}(N, k)=2\left(\left\lfloor\frac{N-k}{2 k}\right\rfloor+\sum_{l=1,3, \ldots}^{\left\lfloor\frac{N}{2 k}\right\rfloor}(2 l k-1)\right) .
\end{aligned}
$$

Se o truncamento descrito anteriormente for considerado, a vantagem computacional desta segunda abordagem torna-se maior ainda. Nesse caso, o número de multiplicações, $M_{2, T}$, e o de adições, $A_{2, T}$, valem

$$
\begin{array}{r}
M_{2, T}(k)=2, \\
A_{2, T}(N, k)=4 k-2 .
\end{array}
$$

\section{AnÁlise Espectral Não-Uniforme Via AFT}

Nesta seção, a aplicação da NDFT via AFT à análise espectral não-uniforme é discutida. Focaliza-se uma situação em que se pretende verificar, com determinada precisão, a presença de freqüências específicas num sinal. Assume-se que os tons a serem detectados não estão espaçados de maneira uniforme ao longo do espectro. Portanto, o uso de uma DFT com comprimento prático não provê a resolução necessária. Este é o motivo que torna essencial o emprego da NDFT, por exemplo, na detecção de sinais dual-tone multifrequency (DTMF) [3], cujo cenário é usado como referência na descrição a seguir.

Um sinal DTMF ideal é composto pela soma de dois tons senoidais. Um desses tons pertence a um grupo de frequiências baixas $(697 \mathrm{~Hz}, 770 \mathrm{~Hz}, 852 \mathrm{~Hz}$ e $941 \mathrm{~Hz})$, associado às linhas do teclado DTMF; o outro pertence a um grupo de frequiências altas $(1209 \mathrm{~Hz}, 1336 \mathrm{~Hz}, 1477 \mathrm{~Hz}$ e $1633 \mathrm{~Hz})$, associado às colunas do mesmo teclado [3]. Cada combinação específica entre uma frequiência baixa e uma alta representa um dos 16 símbolos presentes no teclado $(0-9, \mathrm{~A}-\mathrm{D}, *, \#)$.

\section{A. Componentes Espectrais}

Dentre os diversos requerimentos necessários à concepção de um detector DTMF comercial, a capacidade de avaliar a energia associada a cada uma das 8 freqüências padrão é de fundamental importância [3]. Esse é o aspecto que é tratado no presente trabalho. A execução dessa tarefa requer, inicialmente, a determinação do tamanho das janelas a serem usadas na aplicação do algoritmo. Nesse contexto, mostra-se que janelas de comprimento $N_{L}=212$, para as freqüências baixas, e $N_{H}=106$, para as altas, atendem às exigências relacionadas à duração e à variação sobre as freqüências operacionais do sinal recebido [9]. Daí, aplicando o mesmo procedimento do exemplo apresentado na Seção III-A, determina-se a componente espectral associada a cada freqüência. A componente referente a $f=697 \mathrm{~Hz}$, por exemplo, é

$$
k-\beta=697 \frac{212}{8000}=18,47,
$$

ou seja $k=19$ e $\beta=0,53$.

\section{B. Médias Alternantes}

Considere-se a detecção da freqüência $f=697 \mathrm{~Hz}$ pela aplicação da AFT. Usando a Equação (6), para $k=19$ e $N=212$, verifica-se que as seguintes médias alternantes de Bruns são necessárias ao cálculo de $R[19]$ :

$$
\tilde{B}_{38}(0), \tilde{B}_{114}(0), \tilde{B}_{190}(0) \text {. }
$$

Analogamente, o cálculo de $I[19]$ requer as médias:

$$
\tilde{B}_{38}\left(\frac{1}{76}\right), \tilde{B}_{114}\left(\frac{1}{228}\right), \tilde{B}_{190}\left(\frac{1}{380}\right) \text {. }
$$

Cada uma das médias requeridas é calculada aplicando-se ao sinal que se deseja analisar a Equação (5), se a primeira abordagem for utilizada, e a Equação (14), caso se opte pela segunda abordagem. Neste ponto, observa-se que amostras do sinal em instantes de tempo discreto não-inteiros são necessárias. Uma vez que tais amostras não estão disponíveis, procedimentos de interpolação são requeridos para que as mesmas sejam estimadas.

Em [8], técnicas para interpolação em transformadas aritméticas são propostas. Entre elas, destaca-se a de interpolação ideal, que permite obter o valor exato do sinal no respectivo instante de tempo discreto não-inteiro. Naturalmente, a aplicação deste procedimento eleva consideravelmente o custo computacional da AFT. Como a principal vantagem das transformadas aritméticas é a baixa complexidade, quando comparada aos métodos usuais para o cálculo de transformadas discretas, nos resultados apresentados a seguir, opta-se por utilizar apenas interpolações de ordem um e de ordem zero para estimar as amostras não disponíveis. Na primeira opção, cada amostra a ser estimada requer uma multiplicação e uma adição extras; na interpolação de ordem zero, nenhuma operação aritmética extra é necessária, uma vez que, simplesmente, utiliza-se a amostra disponível mais próxima. 


\section{Simulações e Resultados}

A implementação das duas abordagens desenvolvidas para o cálculo da NDFT via AFT foi realizada no Matlab ${ }^{\circledR}$. Para fins de comparação, o cálculo através da Equação (9), que seria efetuado pelo algoritmo de Goertzel, foi também implementado. Para cada uma das freqüências DTMF, foi gerado um sinal discreto na forma

$$
x[i]=\cos \left(\frac{2 \pi f i}{f_{s}}\right), i=0,1, \ldots, N-1,
$$

onde $f_{s}=8000 \mathrm{~Hz}$, ao qual os algoritmos mencionados foram aplicados visando à obtenção da componente $X[k]$ de interesse.

No caso particular da detecção da freqüência $f=697 \mathrm{~Hz}$, foi gerado um sinal na forma da Equação (17), com $N=212$. O valor de $|X[19]|^{2}, \beta=0,53$, foi então computado via algoritmo de Goertzel, $\mathrm{AFT}_{1}, \mathrm{AFT}_{1, T}, \mathrm{AFT}_{2}$ e $\mathrm{AFT}_{2, T}$ (usando interpolações de ordem um e de ordem zero). Na Tabela I, os resultados obtidos são apresentados. Tomando como referência o algoritmo de Goertzel, verifica-se que a diferença entre o valor de $|X[19]|^{2}$ computado por uma AFT e aquele obtido por sua versão truncada é desprezível. Esse resultado se mantém para os outros tons DTMF. Assim, no cenário considerado, constata-se a validade de utilizar apenas os algoritmos $\mathrm{AFT}_{1, T}$ e $\mathrm{AFT}_{2, T}$, os quais, conforme exposto na seção anterior, demandam complexidade aritmética mais baixa.

TABELA I

VALORES DE $|X[19]|^{2}$ OBTIDOS PELA APLICAÇão DE DIVERSOS ALGORITMOS AO SINAL $x[i]\left(f=697 \mathrm{~Hz}, N=212, f_{s}=8000 \mathrm{~Hz}\right)$.

\begin{tabular}{l|c|cc|cc}
\hline & Goertzel & $\mathrm{AFT}_{1}$ & $\mathrm{AFT}_{1, T}$ & $\mathrm{AFT}_{2}$ & $\mathrm{AFT}_{2, T}$ \\
\hline Int. Ordem 1 & 106,13 & 103,33 & 103,20 & 103,37 & 103,37 \\
Int. Ordem 0 & 106,13 & 104,85 & 104,53 & 105,50 & 104,66 \\
\hline
\end{tabular}

Calcular o valor de $|X[k]|^{2}=R[k]^{2}+I[k]^{2}$ permite avaliar a energia associada à respectiva freqüência física $f$. Este procedimento equivale a aplicar ao sinal sendo analisado um filtro passa-faixa em que $f$ é a freqüência central, ou seja, a frequiência que recebe maior ganho. Baseando-se nessa interpretação, é possível traçar curvas nas quais se observa a resposta às freqüências em torno de $f$ e comparar as diversas alternativas abordadas ao longo deste trabalho. Com esse propósito, para cada sinal com freqüência na faixa $f=$ $697 \pm 200 \mathrm{~Hz}$ (com passo igual a $1 \mathrm{~Hz}$ ), o valor de $|X[19]|^{2}$ foi computado. Os resultados obtidos estão ilustrados nas curvas das Figuras 1 e 2. Além disso, apresenta-se na Tabela II o número de multiplicações e o de adições necessários à obtenção de $X[19]$.

\section{Discussão}

Na Figura 1, onde se utiliza interpolação de ordem um, verifica-se que a curva obtida pela $\mathrm{AFT}_{1, T}$ está, praticamente, superposta àquela obtida pelo algoritmo de Goertzel. $\mathrm{O}$ único aspecto em que se observa alguma disparidade é a altura do lóbulo principal, que, devido ao truncamento (vide Tabela I), é ligeiramente menor, tanto para a $A F T_{1, T}$ quanto para a
TABELA II

COMPLEXIDADE ARITMÉTICA ENVOLVIDA NO CÁLCULO DA COMPONENTE $X[19]\left(f=697 \mathrm{~Hz}\left(N=212, f_{s}=8000 \mathrm{~Hz}\right)\right.$.

\begin{tabular}{l|c|c|c|c|c}
\hline Interpolação & - & \multicolumn{2}{|c|}{ Ord. 1 } & \multicolumn{2}{c}{ Ord. 0 } \\
\hline & Goertzel & $\mathrm{AFT}_{1, T}$ & $\mathrm{AFT}_{2, T}$ & $\mathrm{AFT}_{1, T}$ & $\mathrm{AFT}_{2, T}$ \\
\hline \# Multiplic. & 216 & 308 & 78 & 156 & 2 \\
\# Adições & 426 & 302 & 150 & 150 & 74 \\
\hline
\end{tabular}

$A F T_{2, T}$. A curva obtida por essa última abordagem também é bastante semelhante àquela gerada via algoritmo de Goertzel. Nesse caso, destaca-se a largura dos lóbulos secundários que, devido ao ajuste aplicado à freqüência de amostragem, são ligeiramente mais estreitos (basta observar os pontos em que a curva pontilhada passa pelo zero).

Na Figura 2, onde interpolação de ordem zero é empregada, as tendências da Figura 1 são mantidas. Adicionalmente, verifica-se, para as duas abordagens utilizadas, à medida que a frequiência se afasta da frequiência central, uma gradativa disparidade em relação à curva obtida pelo algoritmo de Goertzel. Todavia, uma vez que o formato do lóbulo principal é mantido, a análise com precisão na freqüência de interesse não é comprometida.

Em termos de complexidade aritmética, a alternativa que merece maior destaque é a que usa a $\mathrm{AFT}_{2, T}$ com interpolação de ordem zero. Ao custo de apenas duas multiplicações e de um número de adições que é menos da metade do requerido por outras alternativas, essa implementação é capaz de prover resultados satisfatórios, como se pode avaliar nas curvas expostas. Naturalmente, quando se deseja analisar um conjunto específico de frequiências, combinações entre as opções propostas são válidas, uma vez que a sensibilidade às interpolações realizadas deve variar. Isso pode ser visualizado na Figura 3, onde se ilustra a aplicação da $\mathrm{AFT}_{2, T}$, com interpolações de ordem um e zero, à análise de $f=1209 \mathrm{~Hz}$ $\left(N=106, f_{s}=8000 \mathrm{~Hz}\right)$. Para essa freqüência, tem-se

$$
k-\beta=1209 \frac{106}{8000}=16,02 \text {, }
$$

ou seja $k=17$ e $\beta=0,98$. Na referida figura, percebe-se que a diferença entre as curvas obtidas via AFT e a obtida usando o algoritmo de Goertzel é mais aparente. Isso acontece porque, quanto mais elevada a freqüência de um sinal, para uma freqüencia de amostragem específica, maiores são os erros cometidos na estimativa de amostras em instantes de tempo não-inteiros.

\section{CONCLusões}

Este trabalho propõe um novo procedimento para a realização de análise espectral não-uniforme, baseado na transformada aritmética de Fourier. A partir da teoria desenvolvida e dos resultados discutidos, é possível concluir que a AFT é uma ferramenta com alto potencial de aplicação. Por oferecer diversas alternativas de implementação, esse algoritmo é capaz de se adaptar a requisitos de precisão e de custo distintos. Nesse sentido, a forma como a amostragem espectral nãouniforme é realizada e a ordem da interpolação empregada 


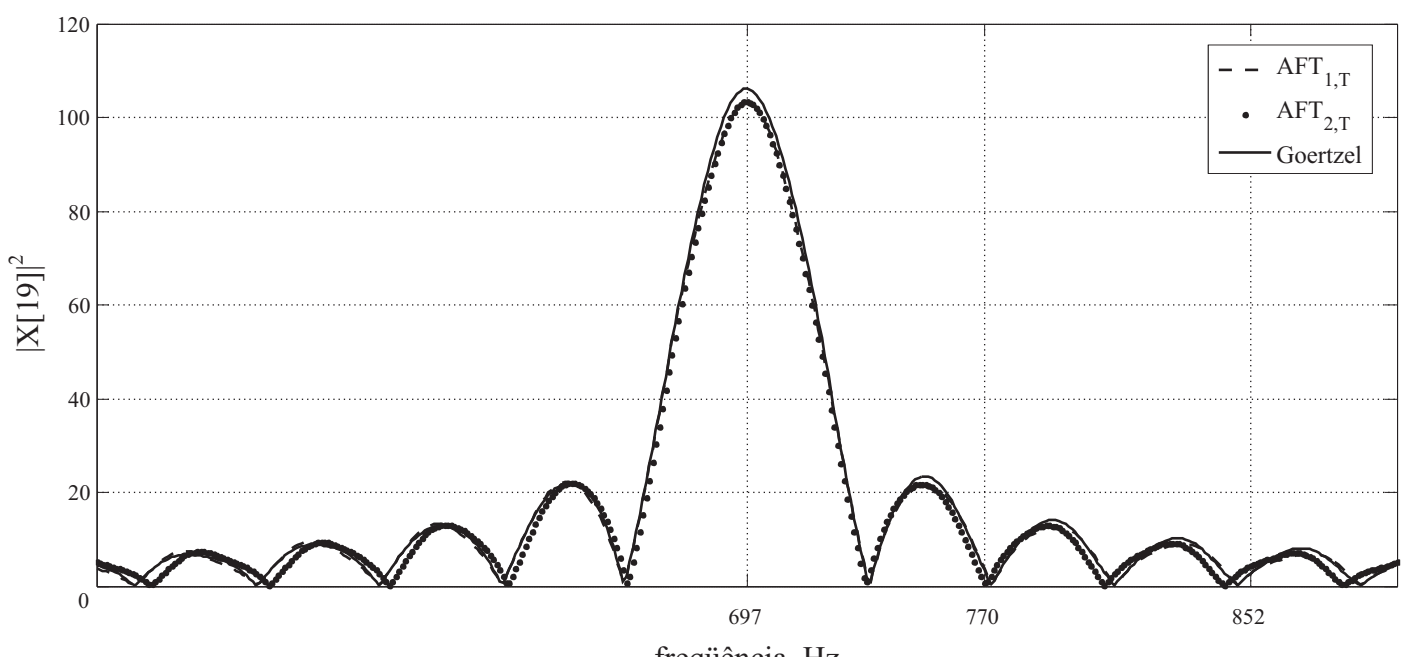

freqüência, $\mathrm{Hz}$

Fig. 1. Resposta em freqüência (magnitude ao quadrado) para análise de $f=697 \mathrm{~Hz}$ (interpolação de ordem um).

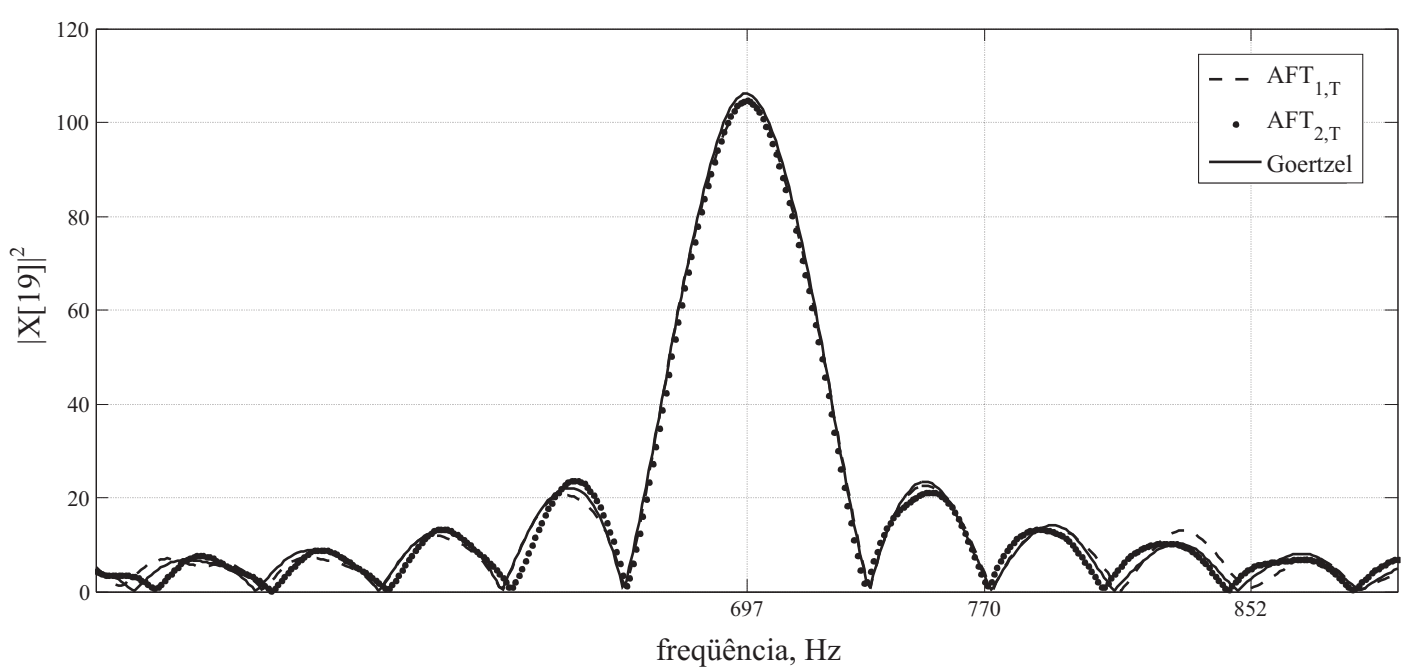

Fig. 2. Resposta em freqüência (magnitude ao quadrado) para análise de $f=697 \mathrm{~Hz}$ (interpolação de ordem zero).

são aspectos de fundamental importância. Tais características devem ser escolhidas de modo a balancear o número de operações aritméticas necessárias e a diferença em relação ao resultado da NDFT implementada por métodos usuais.

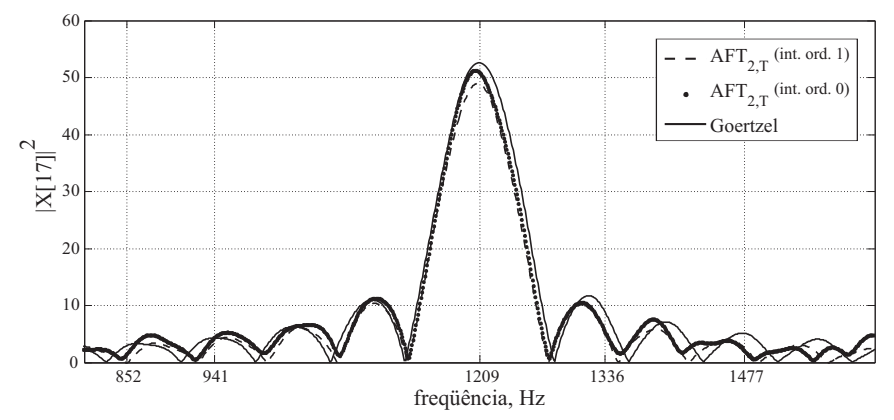

Fig. 3. Resposta em freqüência (magnitude ao quadrado) para análise de $f=1209 \mathrm{~Hz}$ (interpolações de ordem um e zero).

\section{REFERÊNCIAS}

[1] A. V. Oppenheim, R. W. Schafer, and J. R. Buck, Discrete-Time Signal Processing, Prentice Hall, 2nd edition, 1999.

[2] S. Bagchi and S. K. Mitra, "The nonuniform discrete Fourier transform and its applications in filter design: part I-1-d," IEEE Trans. on Circuits and Systems II, vol. 43, pp. 422-433, June 1996.

[3] S. Bagchi and S. K. Mitra, "Efficient robust DTMF decoding using the subband NDFT," Signal Processing, vol. 56, pp. 255-267, Feb. 1997.

[4] I. S. Reed, M. T. Shih, T. K. Truong, E. Hendon, and D. W. Tufts, "A VLSI architecture for simplified arithmetic Fourier transform algorithm," IEEE Trans. Signal Processing, vol. 40, pp. 1122-1133, May 1992.

[5] J. B. Lima, R. M. Campello de Souza, H. M. de Oliveira, and M. M. Campello de Souza, "Faster DTMF decoding," in International Conference on Telecommunications, J. N. Souza, P. Dini, and P. Lorenz, Eds., Berlin, 2004, Lecture Notes in Computer Science, pp. 510-515, Springer.

[6] Z. Xianchao, H. Liusheng, and C. Guoliang, "A new approach for computing the discrete Fourier transform of arbitrary length," in Proceedings of ICSP 2000, Aug. 2000, pp. 81-84.

[7] H. Bruns, Grundlinien Wissenschaftlichichnen Rechnens, B. G. Teubner Verlag, Leipzig, Germany, 1903.

[8] R. J. S. Cintra and H. M. de Oliveira, "A short survey on arithmetic transforms and the arithmetic Hartley transform," Journal of the Brazilian Telecommunications Society, vol. 19, no. 2, pp. 68-79, Aug. 2004.

[9] M. D. Felder, J. C. Mason, and B. L. Evans, "Efficient dual-tone multifrequency detection using the nonuniform discrete Fourier transform," IEEE Signal Processing Letters, vol. 5, pp. 160-163, July 1998. 